\title{
Frontières
}

\section{André Bergeron et Éric Volant, Le suicide et le deuil, Montréal, Éditions du Méridien, 1998, 124 pages}

\section{Lucie Charbonneau et Isabelle Martineau}

Volume 12, numéro 1, automne 1999

URI : https://id.erudit.org/iderudit/1074526ar

DOI : https://doi.org/10.7202/1074526ar

Aller au sommaire du numéro

\section{Éditeur(s)}

Université du Québec à Montréal

\section{ISSN}

1180-3479 (imprimé)

1916-0976 (numérique)

Découvrir la revue

Citer ce compte rendu

Charbonneau, L. \& Martineau, I. (1999). Compte rendu de [André Bergeron et Éric Volant, Le suicide et le deuil, Montréal, Éditions du Méridien, 1998,

124 pages]. Frontières, 12(1), 119-120. https://doi.org/10.7202/1074526ar d'utilisation que vous pouvez consulter en ligne.

https://apropos.erudit.org/fr/usagers/politique-dutilisation/ 
portant sur Le suicide et le deuil. II n'est pas dans nos habitudes de critiquer les livres de nos collègues. Cependant, la détresse occasionnée par certains passages de ce livre chez de nombreuses personnes endeuillées par suicide, nous a convaincues de rédiger cette mise en garde. Les auteurs s'adressent aux personnes endeuillées par suicide et à leurs proches, mais aussi aux professionnels de la santé, travailleurs sociaux, enseignants, directeurs de maisons funéraires, membres du clergé, bénévoles, etc. (p 1 et 2). Ils visent deux objectifs : comprendre les phénomènes du deuil et du suicide et aider la personne endeuillée à prévenir ou résorber une potentielle crise suicidaire (p. 4).

Ce livre propose un ensemble de textes liés à la question du deuil et du suicide qui, bien qu'il n'apporte rien de nouveau, offre l'avantage d'un format de poche peu dispendieux. Pour la question du deuil et particulièrement $d u$ deuil par suicide, le livre de Monique Séguin et Lucie Fréchette (1995), Le Deuil, semble plus intéressant et complet. Mais si ce n'était que cela, nous arrêterions ici notre commentaire.

En effet, toute personne travaillant en prévention du suicide ne peut que se réjouir de la parution d'un tel livre. Il y a un manque flagrant de matériel écrit, accessible à Monsieur et à Madame tout le monde portant sur cette délicate question qu'est le deuil d'une personne décédée par suicide. Malheureusement, il n'y a pas lieu de se réjouir.

Dans ce livre, les auteurs tiennent des propos qui ne traduisent pas l'état actuel des connaissances. À la page 57 , ils indiquent que les filles ont une plus grande incidence de "suicides ratés". II y a déjà plusieurs années que tous les groupes et les associations travaillant à la prévention du suicide demandent de bannir du vocabulaire les termes "suicide raté» ou "suicide réussi». Cette demande n'est pas fortuite. En effet, les termes "rater» et "réussir» sont porteurs de sens. "Rater» signifie vivre un échec et réussir signifie vivre un succès. Ainsi, selon ces termes, la personne qui ne meurt pas suite à sa tentative de suicide serait dans une situation d'échec alors que celle qui en décède se trouverait dans une situation de succès. Ce qui n'est évidemment pas le cas. Il faut plutôt utiliser les termes "tentative de suicide» pour les actes suicidaires ne menant pas à la mort et «suicide complété» pour les actes létaux.

A la page 60 , ils écrivent que le suicide "dérive de la capacité humaine de mourir par choix». Mais le suicide est-il vraiment un choix? N'est-ce pas la seule avenue perceptible à un être humain aveuglé par la souffrance? Le suicide ne serait-il pas plutôt une absence de choix? La personne suicidaire ne voit plus de solution pour mettre fin à sa souffrance. Après avoir fait appel à toutes ses ressources, le suicide représente souvent la seule avenue envisageable. Ce n'est pas là un choix.

Sur cette même page, les auteurs affirment que "On admet aujourd'hui de plus en plus que seulement $10 \%$ des suicidés souffrent de maladie mentale». Certaines études scientifiques indiquent qu'entre $80 \%$ et $90 \%$ des personnes suicidées souffraient d'une maladie mentale, selon le DSM IV, au moment de leur décès. Bien que nous devions nous questionner sur l'étiologie des maladies mentales et donc de leur rôle dans la genèse des comportements suicidaires, force est de constater qu'il existe un lien entre le suicide et la maladie mentale.

De plus, rappelons que ce livre s'adresse aux endeuillés. Ces personnes ont perdu un être cher par suicide. Comme le soulignent les docteurs É. Volant et A. Bergeron, les endeuillés passent pas plusieurs émotions difficiles dont la honte et la culpabilité. Cependant, les auteurs présentent dans leur livre une grille pour dépister le risque suicidaire chez les jeunes (p. 86-95). Les endeuillés risquent fort $d^{\prime} y$ découvrir plusieurs signes laissés par la personne suicidée et qu'ils n'ont pas su interpréter. Ce constat ne pourra qu'exacerber leur sentiment de culpabilité.

Malheureusement, en s'adressant à la fois aux endeuillés et aux intervenants, les auteurs visent une cible large sans l'atteindre. En effet, les endeuillés veulent des réponses à leurs questions et, surtout, ils recherchent une validation de leur souffrance tandis que les intervenants sont en quête d'outils d'intervention.

Mais ici encore, si ce n'était que de cela, le livre Le suicide et le deuil pourrait tout de même avoir sa place dans les librairies. La goutte qui fait déborder le vase se trouve à la page 75 . Les auteurs écrivent, en parlant de l'intervenant qui travaille auprès de personnes endeuillées, potentiellement suicidaires : "Dans certains cas sans doute exceptionnels, il pourrait aller jusqu'à assister la personne en l'aidant à créer les conditions les plus favorables pour poser son geste ou en l'accompagnant dans ses derniers moments.» Que veuton dire ici? Dans cette phrase, on retrouve trois éléments: le jugement, l'assistance et l'accompagnement. Analysons ces éléments.

Le jugement: selon les auteurs, l'intervenant, avec l'appui d'autres intervenants (p. 76), doit évaluer la condition de la personne suicidaire et juger s'il peut l'aider à passer à l'acte de la manière la plus favorable possible. Sur quels critères portera-t-il son jugement? II jugera d'abord, selon ses valeurs propres, que la vie de cette personne est trop souffrante et sans issue. II considérera ensuite si la personne est en mesure de faire un choix éclairé et rationnel. Après avoir écouté le récit de vie de la personne et analysé avec elle les autres avenues, il conclura, avec elle, que le suicide est la seule voie possible.

Peut-on cependant parler d'un choix rationnel chez une personne en crise suicidaire? Dans l'une de ses conférences, Brian Mishara (professeur à I'UQAM et directeur du Centre de recherche et d'intervention sur le suicide et l'euthanasie) remarquait que très peu de décisions majeures de nos vies sont prises sur des bases rationnelles. Le choix d'un conjoint, la venue d'un enfant, l'achat d'une maison se font le plus souvent sur un coup de cœur. II se demandait alors comment une personne souffrante, souvent désespérée, pouvait être apte à prendre une décision rationnelle sur une question aussi importante que de mettre fin à ses jours.

L'assistance: que veut-on dire ici par «créer les conditions les plus favorables pour poser son geste"? Cela veut dire que si l'intervenant juge, malgré tout, que la personne a fait un choix éclairé, il peut l'aider à passer à l'acte de la manière la plus efficace possible. Rappelons que sur le plan juridique, au Canada, conformément à l'article 224 du Code criminel, celui qui aide une personne à se donner la mort l'encourage ou la conseille est passible de 14 ans de prison. Cette loi vise, entre autres, à protéger contre l'incitation au suicide collectif, ou encore à protéger celui qui serait dans un état dépressif et dont on pourrait abuser.

L'accompagnement: il y a plusieurs façons d'accompagner une personne suicidaire. Les plus utilisées consistent à écouter la personne, la soutenir dans sa recherche d'alternatives, tenter d'abaisser son niveau de souffrance et, le cas échéant, appeler 911 pour éviter 
un passage à l'acte. Les auteurs proposent une façon ultime: accompagner la personne dans ses derniers moments. Pourquoi celle-ci plutôt qu'une autre?

L'intervenant pourrait proposer à la personne des façons alternatives de gérer sa souffrance. À la limite, il pourrait l'encourager à prendre une longue cuite au champagne ou encore l'inviter pour une fin de semaine de camping dans les Laurentides. Ces formes d'accompagnement paraissent farfelues? Pourquoi le seraient-elles davantage que d'accompagner la personne dans ses derniers moments ? Ici, du moins, la personne suicidaire risque de voir sa souffrance diminuer légèrement et peut-être suffisamment pour vouloir continuer de vivre.

Loin de nous l'idée de demander aux intervenants de s'impliquer trop personnellement avec leurs clients. Cependant, l'accompagnement que proposent les auteurs de Le suicide et le deuil, démontre un fatalisme et une acceptation du suicide qui s'avèrent plus que dangereux. Pourquoi? Parce que ce livre s'adresse aussi aux endeuillés.

Jamais nous n'aurions écrit cette critique, si ce n'étaient des commentaires désemparés de personnes endeuillées par le suicide d'un fils, d'une amie, d'une sœur. Ils ont vu dans les propos des auteurs un acquiescement à l'option suicide. Cette option, ils l'ont parfois envisagée et ils la craignent. C'est ce qui pousse certains endeuillés à consulter: la peur de passer à l'acte. Et voilà que les auteurs du livre dans lequel ils ont cherché réconfort et réponses, leur ouvre l'option du suicide. En laissant cette place à l'«option suicide», les auteurs de Le suicide et le deuil contribuent à cautionner cette option dans la société québécoise.

Lors d'une conférence, le docteur Mounir Sami de l'hôpital de Montréal pour enfants, rappelait que dans les cultures qui rejettent l'option suicide, il y a effectivement moins de suicides. Comme société, le Québec doit dire NON à l'option suicide. Non, parce que le suicide est en hausse constante. Non, parce que le suicide s'attaque de plus en plus à la jeunesse. Non, parce que le suicide nous prive de parents, de collègues, d'amis. Non, parce que le suicide n'est pas un choix, mais un manque de choix. Non, parce que le suicide brise nos vies. Non, parce qu'il est possible de soulager la souffrance et de redonner un sens à la vie. Non, parce qu'il est possible de prévenir le suicide.
Il est certain que les auteurs ont voulu bien faire. Cependant l'expérience vécue sur le terrain, auprès des endeuillés et des personnes souffrantes indique que ce livre peut avoir des effets néfastes. II faudrait donc revoir son contenu avant de le placer dans les mains de personnes endeuillées.

Lucie Charbonneau avec la collaboration d'Isabelle Martineau 